\title{
Review of Spinal Pedicle Screws
}

\author{
Bahaa Ali Kornah, Faisal Hasan Zayed, Ahmed Kotb Ahmed Elkomy* \\ Department of Orthopedic Surgery, Faculty of Medicine, Al-Azhar University \\ *Corresponding author: Ahmed Kotb Ahmed Elkomy, Email: anakonda100098@ gmail.com, Mobile: (+20) 01552990033
}

\begin{abstract}
Background: Pedicle screws are medical implants which are implanted posteriorly into the vertebrae of the spine and longitudinally connected to a rod to form a construct which corrects spinal alignment or promotes stabilization. Pedicle screw fixation is considered to be the gold standard of spinal internal fixation due to its many benefits in a variety of debilitating spinal conditions. Objective: The aim of the work is to evaluate the use of spinal pedicle screws for different lesions in the past, in the present and in the future. Conclusion: Some successful applications of pedicle screws include surgical treatment of scoliosis reduction and stabilization of spondylolisthesis, stabilization of unstable spinal fractures, simple lumbar degenerative disc disease, primary or metastatic tumors of the lumbar spine, and surgical revision of symptomatic pseudoarthrodesis of the lumbar region.
\end{abstract}

Keywords: Spinal, Pedicle Screws.

\section{INTRODUCTION}

Pedicle screws are medical implants which are implanted posteriorly into the vertebrae of the spine and longitudinally connected to a rod to form a construct which corrects spinal alignment or promotes stabilization. Pedicle screw fixation is considered to be the gold standard of spinal internal fixation due to its many benefits in a variety of debilitating spinal conditions ${ }^{(\mathbf{1})}$. The first use of pedicle screws in 1959 has been credited to Boucher who used a longer facet screw across the pedicle ${ }^{(2)}$.The first deliberate attempt to put pedicle screws through the isthmus of the pedicle was done by Harrington and Tullos ${ }^{(3)}$. The plates used by Roy-Camille in 1979 had a fixed screw-hole distance for application ${ }^{(4)}$.

In contrast, Gaines et al. developed the variablescrew placement (VSP) plate, which allowed better placement of pedicle screws according to individual patient anatomy and provided greater clinical latitude in comparison to the Roy- Camille plate ${ }^{(\mathbf{1}, \mathbf{4})}$. This technology is now almost exclusively used when securing fusion constructs in the thoracolumbar spine, due to the purported improved fusion rates and rigidity afforded by these constructs. Furthermore, studies have found that pedicle screws are biomechanically advantageous when compared to predecessors, including previous rod and hook systems ${ }^{(5)}$.

\section{AIM OF THE WORK}

The aim of the work is to evaluate the use of spinal pedicle screws for different lesions in the past, in the present and in the future.

\section{Basic Spinal Anatomy}

Important Structures:

The important components of the lumbar spine include

- Bones and joints.

- Connective tissues.

- Muscles.

- Blood Supply

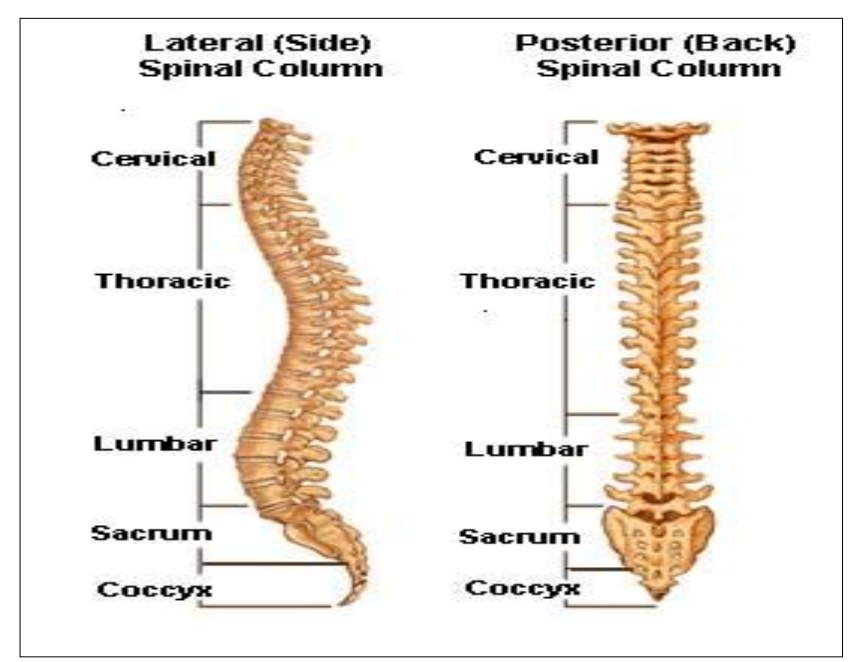

Figure (1) General outline of the spine ${ }^{(6)}$.

\section{Bones and Joints}

\section{The Vertebral Column}

Originating at the cranium, the human vertebral column consists of a series of 33 inferiorly extending vertebrae divided into five sections. The superior most section of the vertebral column is the cervical which consists of seven vertebrae that make up the neck, followed by twelve vertebrae in the thoracic, five in the lumbar, and five and four fused vertebrae making up the sacrum and coccyx, respectively.

Each of the cervical, thoracic, and lumbar vertebrae are separated by intervertebral discs, which allow for flexibility of the spine. In general, due to the larger loads that are placed on inferior vertebrae as opposed to those more superior, the size of each individual vertebra increases inferiorly down the spine until reaching L5 and then decreases again at the sacrum and coccyx ${ }^{(7)}$.

\section{Thoracic vertebrae:}

All 12 thoracic vertebrae support ribs and thus show facets for the articulations of these structures. The first and last four have peculiar manner of costal 
articulations, but the second to eighth may be covered by a common description ${ }^{\left({ }^{(8)}\right.}$.

\section{Lumbar vertebrae:}

There are five lumbar vertebrae, each vertebra has three functional components: the vertebral bodies, designed to bear weight; the neural arches, designed to protect the neural elements; and the bony processes (spinous and transverse), designed as outriggers to increase the efficiency of muscle action ${ }^{(9)}$.

\section{The Facet Joints:}

The zygapophyseal joints are synovial joints that permit simple gliding movements. Although the lax capsule of the joints is supported to some extent from anteriorly by the ligamentum flavum, and posteriorly by supraspinous ligament, the major structure restraining movement in these joints are the outermost fibres of the annulus fibrosus.

When these annulus fibres exhibit degenerative changes, excessive joint movement is permitted. This is why degenerative changes within the discs render the related posterior joints vulnerable to strain ${ }^{(\mathbf{1 0})}$.

\section{The Neural Foramen:}

On the left and right side of each vertebra is a small tunnel called neural foramen. The two spinal nerves that leave the spine at each vertebra go through the foramina, one on the left and one on the right. Each nerve branches in and just beyond the foramen into ventral and dorsal rami.

The dorsal rami supply the facet joints as mentioned above. Besides, there is the recurrent meningeal (sinuvertebral nerve) which may be best considered as recurrent branches of the ventral rami (10).

\section{Design of Pedicle Screw}

Much needs to be considered when determining the proper pedicle screw design to be used for spinal fusion in an osteoporotic patient. Increasing the diameter and length of the screw has the potential to produce larger pullout forces, but they also increase the risk of fracturing the surrounding fragile bone $^{(11)}$.

Tapering the diameter of the screw is believed to help compress surrounding bone, which may in turn enhance the screw's fixation in the vertebra (11). Different thread designs serve a diverse range of mechanical functions that must take into consideration the material properties of the bone that it is to be paired with ${ }^{(12)}$.

Moreover, a screw material that would offer not only excellent mechanical properties but also exceptional biocompatible properties is crucial for successful long term performance ${ }^{(\mathbf{1 3}) \text {. }}$

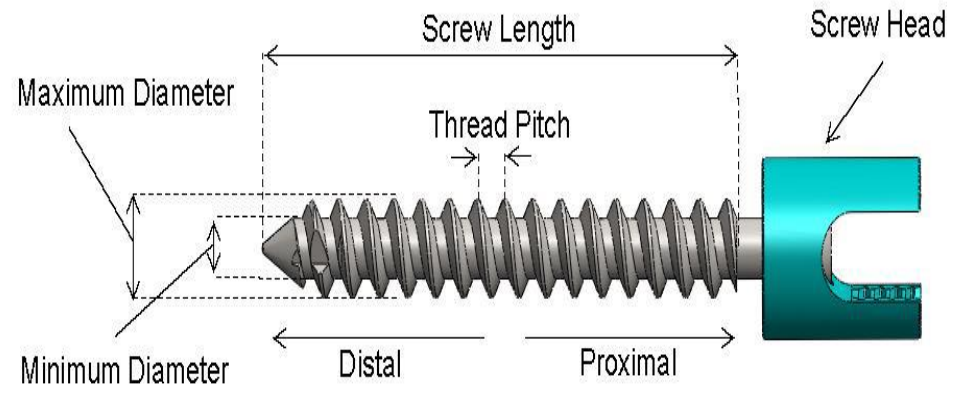

Figure (2): Standard pedicle screw ${ }^{(13)}$.

\section{Advantages with the use of pedicle screws}

The increasing use of transpedicular fixation devices in preference to rods, hooks, and wires is because they offer rigid segmental fixation after decompression and arthrodesis. They have the ability to control all the three columns of the spine from a posterior approach and possibly reduce the number of segments that need to be fused ${ }^{(14)}$. This allows segmental instrumentation i.e. limiting the instrumentation to one or two motion segments, thus preserving maximum motion. Proper use of the pedicle screw obviates canal intrusion, and since this system does not depend on the facet or laminae for their attachment, it allows for preservation of mobile segments above and below the level of affected spine segments. Moreover, screw rod or screw plate constructs can be used to apply selective forces like compressing, rotating, distracting, lordosing, etc., depending upon the clinical situation. Such rigid internal fixation also allows for earlier mobilization in the postoperative period. Studies have shown that since pedicle screw constructs are more rigid in comparison to traditional forms of internal fixation, they have a higher rate of successful fusion ${ }^{(\mathbf{1 5})}$.

\section{Indications of pedicle screw fixation}

- Pursuant to the FDA's ruling in 1998, the safety and effectiveness of pedicle screw spinal systems have been established only for spinal conditions with significant mechanical instability or deformity requiring fusion with instrumentation ${ }^{(14)}$. Some successful applications of pedicle screws include (16):-

- Surgical treatment of scoliosis and adult degenerative lumbar scoliosis. Better results were observed with the use pedicle screw for corrections in the coronal and sagittal planes, and correction of tilt-angle and rotations ${ }^{(17)}$.

- Reduction and stabilization of spondylolisthesis, and following laminectomy with improved ability to reduce and maintain reduction of higher-grade slips ${ }^{(17)}$.

- Stabilization of unstable spinal fractures, like burst fractures as well as treatment of posttraumatic kyphosis ${ }^{(\mathbf{1 8})}$. 
- Simple lumbar degenerative disc disease with union rates of about $95 \%$ for two and three-level lesions ${ }^{(\mathbf{1 9})}$.

- Correction, stabilization, and maintenance of correction of spina bifida and postlaminectomy deformities ${ }^{(20)}$.

- Spinal osteotomy in patients with fixed multiplanar spinal deformities due to ankylosing spondylitis (21) and in those with severe flatback deformity ${ }^{(20)}$.

- Cases of extensive decompression or resection and short-segment treatment of primary or metastatic tumors of the lumbar spine ${ }^{\text {(22) }}$.

- Iatrogenic instability developing after decompressive procedures;

- Surgical revision of symptomatic pseudoarthrodesis of the lumbar region ${ }^{(22)}$.

\section{Complications associated with the use of pedicle screws}

\section{A. Screw misplacement or coupling failure:}

The rate of screw misplacement ranged from $0-25 \%$ in patients with scoliosis and nearly $4.2 \%$ in patients with degenerative diseases. Coupling failure of the device is caused due to inadequate nut tightening, and results in disengagement of the screw from the clamp elements of the $\operatorname{rod}^{(23)}$.

\section{B. Nerve-root injuries:}

A variable rate of nerve-root and/or cauda equina injuries were reported and associated with pain and sensory deficit. Screws that are placed medially and inferiorly are the ones that place the nerve at the risk of injury. Matsuzaki et $\boldsymbol{a l} .{ }^{(24)}$ reported nerve neurological impairment in about $11 \%$ of patients and sensory impairment in $3.5 \%$ out of the total 57 that were reviewed. Prevalence of nerve injury as low as 1-2 \% was reported in different studies. Dural injury was reported in seven of 124 patients $(6 \%)$, and in four of the $4790(0.1 \%)$ pedicle screws. Neurological injuries associated with misplaced screws were reported to be $0-12 \%$ in another study ${ }^{(25)}$.

\section{Breakage or fracture of the screw:}

One study showed that 21 of $74(36 \%)$ screws had fatigue failure. In other studies, the frequency of screw breakage ranged from $0.5-11.2 \%$ of the inserted screws. Lonstein et $_{\boldsymbol{a l}}{ }^{(\mathbf{2 6 )}}$ related screw breakage to three factors: design of the screw, presence of pseudoarthrodesis and their use in burst fractures.

\section{Nonunion or Screw loosening:}

Loosening of pedicle screws indicates micromovement at the region of the screw and rod. Screw loosening was commonly seen in patients with low bone mineral density (BMD) and osteoporosis. In a selected survey of the members of the American
Back Society, screw loosening was observed in $0.81 \%$ of 617 patients, and ranged from $0.6 \%$ to $11 \%$ in the literatures reviewed by Yi et al. ${ }^{(27)}$. Pihlajämaki and coworkers $^{(23)}$, in retrospective study of 102 patients of non-traumatic disorders with posterior lumbar interbody fusion (PLIF) with transpedicular screws fixation, reported radiologic screw loosening in 18 patients (17\%). Loosening was most commonly seen in patients with multilevel instrumentation and in patients with sacral screws ${ }^{(23)}$. Fracture of the pedicle has been reported in about $0.2-4.3 \%$ cases and is more common in patients with lower bone mineral density (BMD). Other complications include bending of screws and infections and injury to the blood vessels (19).

\section{Methods designed to improve fixation of the pedicle screw}

Maintaining the bone-implant interfaces and initial rigidity is a significant challenge. In general, the stability of pedicle screws depends mainly on the geometric characteristics of the screw and on the mechanical properties of the trabecular bone adjacent to the screw. Immediately after surgery, the implants are expected to provide sufficient stabilization to maintain alignment and promote fusion, and are thus subjected to high loads in this immediate postoperative period ${ }^{(28)}$. Inadequate anchorage of the pedicle screws through the pedicle creates increased and prolonged stresses at the bonescrew interface, resulting in osteolysis and implant failure. These conditions have been particularly evident in patients with low bone mineral density (BMD) and osteoporosis, neuromuscular disorders and postradiation therapy. High failure rates due to inadequate fixation strength at the pedicle screw-bone interface are also a significant problem especially in longer lumbar constructs. Other factors that influence the bony purchase and subsequent fixation strength of a pedicle screw include insertion site and technique, depth of penetration, pedicle screw diameter, and screw design characteristics ${ }^{(29)}$.

Some researchers introduced an alteration in the design of the screw. Polly et $\boldsymbol{a l}^{(\mathbf{3 0})}$ found that increasing the diameter of the salvage screw by $2 \mathrm{~mm}$ caused an increase in insertional torque by $8.4 \%$, whereas increasing the length of the screw did not result in any improvements of insertional torque. Skinner et al. (31) biomechanically assessed and compared four designs of pedicle screws. The results showed that the pullout strength of the screw increased with an increase in the major diameter of the screw, whereas an increase in the pitch caused increased amount of displacement prior to failure. The idea of sacral screw fixation using a hollow screw was also proposed.

The hollow screw supposedly increases contact area with the surrounding bone both on the outer and 
inner surface of the screw, potentially improving fixation within trabecular bone. Schramm et al. ${ }^{(32)}$ demonstrated superior pull-out strength with a novel hollow screw when inserted into the trabecular bone of thoracic vertebral bodies. McLachlin et al. ${ }^{(29)}$ also used a novel hollow screw design in a sacral model under cyclic loading and concluded that the hollow screw was less resistant to loosening in comparison with the conventional solid pedicle screw.

Other orthopedic surgeons have applied alteration in the anatomic trajectory of placement of the pedicle screw to attain augmentation. Currently, two popular methods for insertion of pedicles screws are used: straight-forward technique, in which the sagittal trajectory of the screw parallels the superior endplate of the vertebral body, and the anatomic trajectory, which is directed $22^{\circ}$ in the cephalocaudal direction in the sagittal plane and parallels the anatomic axis of the pedicle. Lehman et al. compared the results of the straight-forward technique with the anatomic technique and showed that straight-forward technique resulted in a $39 \%$ increase in maximum insertional torque and a $27 \%$ increase in pull-out strength compared to the anatomic technique ${ }^{(33)}$. Another option to obtain better purchase was proposed in the lumbar spine by Cook et $\mathbf{a l}^{(34)}$. They used pedicle screws which penetrated the anterior cortex of the vertebral body. Although this procedure is associated with a significant risk, it demonstrated to add approximately $30 \%$ to the pull-out of a cancellous screw in the lumbar spine, although a lot of risk is associated with this procedure. The use of laminar hooks concurrently with the pedicle screws in vitro showed an enhancement in the rigidity of pedicle screw fixation. Other novel augmentation methods suggested in the literature include the use of an expansive pedicle screw design, an expandable anchor and an interlocking screw ${ }^{(34)}$.

The use of osteoinductive materials revolutionized spine surgery. One way to establish a mechanical connection between the implant and the host skeleton is regeneration of bone at the interface. The cornerstones for successful bone-healing are biomechanical stability and biological vitality of the bone, which provides an environment in which new bone can form. Many conditions, such as insufficient vascularization, infection, mechanical instability, and systemic diseases like diabetes, osteoporosis and old age can impair this environment ${ }^{(35)}$. The new methods to improve fixation and stability of implants focus on identifying and understanding the factors that control the regenerating potential of the bone. Various biologics were used for enhancement of lumbar fusion surgery over the last decade. One of the most studied and frequently used to modulate bone apposition on the implant surface is by the use of growth factors. Different growth factors are known to improve osteoblast differentiation and matrix mineralization, like platelet-derived growth factor (PDGF), bone morphogenetic proteins (BMPs), insulin-like growth factors (IGF) and basic fibroblast growth factor (FGF). Among them, BMPs are the most intensively studied group and the most promising group of growth factors used in the enhancement of bone repair ${ }^{(35)}$.

\section{SUMMARY}

Pedicle screws are medical implants which are implanted posteriorly into the vertebrae of the spine and longitudinally connected to a rod to form a construct which corrects spinal alignment or promotes stabilization. Pedicle screw fixation is considered to be the gold standard of spinal internal fixation due to its many benefits in a variety of debilitating spinal conditions.

The anatomy of the vertebral pedicle is of particular interest because it is the narrowest region where a pedicle screw passes. The pedicle is typically oval- shaped but has a complex three- dimensional structure which was described as a teardrop.

The first use of pedicle screws in 1959 was credited to Boucher who used a longer facet screw across the pedicle. The first deliberate attempt to put pedicle screws through the isthmus of the pedicle was done by Harrington and Tullos. The plates used by Roy-Camille in 1979 had a fixed screw-hole distance for application.

In contrast, Gaines et al. developed the variable-screw placement plate, which allowed better placement of pedicle screws according to individual patient anatomy and provided greater clinical latitude in comparison to the Roy- Camille plate.

Pedicle screw placement techniques are freehand, fluoroscopy guided and stereotactic navigation.

In the Free-Hand technique the analogous entry sites guided by differential anatomy are utilized for both the thoracic and lumbar spine. In the thoracic spine the entry site is located at the lower border of the superior articular facet, the medial border of the transverse process, and the pars interarticularis form a triangle, the center of which should be targeted for initial entry. In the lumbar spine, the entry site is located at the intersection of the bony confluences of the pars interarticularis, the transverse process, and the mammillary process of the vertebrae that will be instrumented.

Fluroscopy-Guided technique often utilizes a $\mathrm{C}$-arm to take AP and lateral images.

Image-guided or stereostatic technique is a process that was originally accomplished with a preoperative $\mathrm{CT}$ and then surgeon matching of points on the computer-generated image to anatomical points on the patient.

Some successful applications of pedicle screws include surgical treatment of scoliosis reduction and stabilization of spondylolisthesis, stabilization of unstable spinal fractures, simple 
lumbar degenerative disc disease, primary or metastatic tumors of the lumbar spine and surgical revision of symptomatic pseudoarthrodesis of the lumbar region.

\section{REFERENCES}

1. Gaines RW (2000): The use of pedicle- screw internal fixation for the operative treatment of spinal disorders. Journal of Bone and Joint Surgery, 82: 1458-1476.

2. Boucher HH (1959): A method of spinal fusion. J Bone and Joint Surg Br., 41:248-259.

3. Harrington PR, Tullos HS (1969): Reduction of severe spondylolisthesis in children. Southern Med J., 62:1-7.

4. Roy-Camille R, Saillant G, Mazel C (1986): Plating of thoracic, thoracolumbar, and lumbar injuries with pedicle screw plates. Orthop Clin North Am., 17:147-159.

5. Krag MH, Weaver DL, Beynnon BD et al. (1988): Morphometry of the thoracic and lumbar spine related to transpedicular screw placement for surgical spinal fxation. Spine Phila Pa., 13: 27-32.

6. Wong D and Transfeldt E (2007): Muscloskeletal Anatomy, Neuroanatomy and Biomechanics of the lumbar spine. In: Macnab's Backache, $4^{\text {th }}$ Edition, Lippincott Williams \&Wilkins, Pp. 1- 20.

7. Moore KL, Agur AM, Dalley AF (2011): Essential clinical anatomy. 4th ed. 2011 Baltimore, MD: Lippincott Williams \& Wilkins, 28:703725.

8. Harry JH and Mirvis SE (1999): Hyperflexion Injuries, 3rd ed. Baltimore: Williams \& Wilkins, Pp.245-289.

9. Bogduk N, Macintosh JE, Pearcy MJ (1992): A universal model of the lumbar back muscles in the upright position. Spine, 17(8):897-913.

10. Varlotta GP, Lefkowitz TR, Schweitzer M et al. (2011): The lumbar facet joint: a review of current knowledge: part 1: anatomy, biomechanics, and grading. Skeletal Radiol., 40(1):13-23.

11. Burval DJ, McLain RF, Milks R et al. (2007): Primary pedicle screw augmentation in osteoporotic lumbar vertebrae: biomechanical analysis of pedicle fixation strength. Spine, 32(10): 1077-1083.

12. Chao CK, Hsu CC, Wang JL et al. (2008): Increasing bending strength and pullout strength in conical pedicle screws: biomechanical tests and finite element analyses. Journal of Spinal Disorders and Techniques, 21(2): 130 138.

13. Shea TM, Laun J, Gonzalez-Blohm SA et al. (2014): Designs and techniques that improve the pullout strength of pedicle screws in osteoporotic vertebrae: current status. Biomed Res Int., 2014:748-53.

14. Grubb SA, Lipscomb HJ (1992): Results of lumbosacral fusion for degenerative disc disease with and without instrumentation. Two- to five-year follow-up. Spine, 17:349-355.

15. Ransom N, La Rocca SH, Thalgott J (1994): The case for pedicle fixation of the lumbar spine. Spine, 19(23):2702-6.

16. Kotani Y, Cunningham BW, Cappuccino A et al. (1996): The role of spinal instrumentation in augmenting lumbar posterolateral fusion. Spine, 21(3):278-87.

17. Hu SS, Tribus CB, Diab M et al. (2008): Spondylolisthesis and spondylolysis. J Bone Joint Surg Am., 90:656-671.
18. Dai LD (2002): Low lumbar spinal fractures: management options. Injury, 33(7):579- 82.

19. Mac Millan, MM, Cooper R et al. (1994): Lumbar and lumbosacral fusions using Cotrel- Dubousset pedicle screws and rods. Spine, 19:430-434.

20. Rodgers WB, Williams MS, Schwend RM et al. (1997): Spinal deformity in myelodysplasia. Correction with posterior pedicle screw instrumentation. Spine, 22:2435-43.

21. Merloz P, Troccaz J, Vouaillat H et al. (2007): Fluoroscopy-based navigation system in spine surgery. Proc Inst Mech Eng H., 221: 813-820.

22. Tan JS, Singh S, Zhu QA et al. (2008): The effect of cement augmentation and extension of posterior instrumentation on stabilization and adjacent level effects in the elderly spine. Spine, 33(25):2728-40.

23. Pihlajämaki H, Myllynen P, Böstman O (1997): Complications of transpedicular lumbosacral fixation for non-traumatic disorders. J Bone Joint Surg Br., 79-B:1839.

24. Matsuzaki H, Tokuhashi Y, Matsumoto F et al. (1990): Problems and solutions of pedicle screw plate fixation of lumbar spine. Spine, 15:1159-65.

25. Hsu J, Zuckerman JF, White AH et al. (1987): Internal fixation with pedicle screws. In: White AH, Rothman RH, Roy CD, eds. Lumbar Spine Surgery.CV Mosby, St Louis, Pp.322-38.

26. Lonstein JE, Denis F, Perra JH et al. (1999): Complications associated with pedicle screws. J Bone Joint Surg Am., 81(11):1519-28.

27. Yi X, Wang Y, Lu H et al. (2008): Augmentation of pedicle screw fixation strength using an injectable calcium sulfate cement: an in vivo study. Spine, 33(23):2503-9.

28. Tan JS, Singh S, Zhu QA et al. (2008): The effect of cement augmentation and extension of posterior instrumentation on stabilization and adjacent level effects in the elderly spine. Spine, 33(25):2728-40.

29. McLachlin SD, Beaton BJ, Sabo MT et al. (2008): Comparing the fixation of a novel hollow screw versus a conventional solid screw in human sacra under cyclic loading. Spine, 33:1870-1875.

30. Polly DW, Orchowski JR, Ellenbogen RG (1998): Revision pedicle screws. Bigger, longer shims-what is best? Spine, 23:1374-9.

31. Skinner R, Maybee J, Transfeldt E et al. (1990): Experimental pullout testing and comparison of variables in transpedicular screw fixation: a biomechanical study. Spine, 15:195-201.

32. Schramm M, Krummbein S, Kraus H et al. (2003): Anterior vertebral body screw pullout testing with the hollow modular anchorage system- a comparative in vitro study. Biomed Technik (Berl), 48:356-61.

33. Lehman RA, Polly DW, Kuklo TR et al. (2003): Straightforward versus anatomic trajectory technique of thoracic pedicle screw fixation: a biomechanical analysis. Spine, 28:2058-65.

34. Cook SD, Salkeld SL, Whitecloud TS et al. (2000): Biomechanical evaluation and preliminary clinical experience with an expansive pedicle screw design. J Spinal Disord., 13(3):230-236.

35. Termaat MF, Den Boer FC, Bakker FC et al. (2005): Bone morphogenetic proteins. Development and clinical efficacy in the treatment of fractures and bone defects. J Bone Joint Surg Am., 87:1367-78. 